\title{
A Real-Time Spoken-Language System for Interactive Problem Solving
}

\author{
Patti Price and Robert C.Moore \\ SRI International \\ Menlo Park, CA 94025
}

\section{PROJECT GOALS}

The goal of this project, to develop a spoken-language interface to the Official Airline Guide database, is being developed along two overlapping research and development lines: one focussed on an SLS kernel for database query, and the other on the full interactive system.

\section{RECENT RESULTS}

- Evaluation of SRI's NL, SLS, and speech recognition technologies. SRI's February-92 weighted error rate for ATIS class A and D utterances combined was $31.0 \%$ for NL and $45.4 \%$ for SLS. SRI's February-92 SPREC word error rate for all ATIS utterances was $11.0 \%$. For the speaker-independent, closed $5 \mathrm{~K}$ vocabulary CSR task, SRI achieved a $16.6 \%$ word error rate in the verbalized punctuation test and a $17.1 \%$ word error rate in the non-verbalized-punctuation test, using the standard bigram language model.

- Encouraging initial results in a study of verbal repairs in the ATIS MADCOW corpus; the study included analysis and classification of the repairs in the corpus as well as a method using both pattern matching techniques and acoustic evidence to detect and correct the repairs.

- Implementation of RASTA filtering (high-pass filtering in the Log Spectral domain to remove timeinvariant or slow moving linear channel effects) to improve channel robustness.

- Experiments showing differences between spontaneous and read speech and the need for including spontaneous speech in training, development, and test sets.

- Development of infrastructure necessary for dealing with very large vocabularies and language models such as those associated with the CSR corpus.

- Improvement in GEMINI natural-language processing system's linguistic coverage of a 2100-utterance ATIS traning corpus to $92 \%$ syntactic coverage, $77 \%$ semantic coverage.
- Development of algorithms for tracking evolving discourse structure. On the development set of the ATIS corpus, we find these algorithms correctly determine the discourse context with greater than $90 \%$ accuracy.

- Improvements to the template matcher: new templates, improved portability, more linguistic knowledge incorporated, reduced overgeneration, improved context handling, improved coverage of database retrieval component, all without significantly increasing response time, which is still less than a second.

- Analysis of data on human-machine problem solving using our SLS ATIS system: we have analyzed the effect on user satisfaction and system performance of system errors, user experience, and instructions to users. We have also explored trade-offs of time vs.speed and user satisfaction.

- Collection of data (speech, transcriptions, and logfiles) using our SLS ATIS system. To date this includes 72 speakers, 2301 utterances, and 134 scenarios.

- Improvements in the SLS ATIS demo system, including better paraphrasing of system's understanding, easier to read displays, improved system error messages, simpler control of context mechanism.

- Development with MIT of a method for end-to-end evaluation that takes into account the whole interaction; implementation of the method is in progress.

\section{PLANS FOR THE COMING YEAR}

- Fully integrate GEMINI into SRI's ATIS system.

- Explore tighter integration of speech and NL processing for better overall SLS performance.

- Improve speech recognition computational and word-accuracy performance for the ATIS and CSR domains with better models of the channel, speaker, and spontaneous speech effects. 